\title{
Homotopy analysis method for solving nonlinear diffusion equation with convection term
}

\author{
Bewar A. Mahmood *, Saad A. Manaa, Fadhil H. Easif \\ Department of Mathematics, Faculty of Science, University of Zakho, Duhok, Iraq \\ *Corresponding author E-mail: bewar.ahmed@uod.ac
}

Copyright $\odot 2014$ Mahmood et al. This is an open access article distributed under the Creative Commons Attribution License, which permits unrestricted use, distribution, and reproduction in any medium, provided the original work is properly cited.

\begin{abstract}
In this article the homotopy analysis method (HAM) is used to find a numerical solution for the nonlinear diffusion equation with convection term. The numerical results obtained by using this method compared with the exact solution, by solving numerical example shows that (HAM) is accurate and close to the exact solution.
\end{abstract}

Keywords: Homotopy Analysis Method, Nonlinear Diffusion Equation with Convection Term.

\section{Introduction}

Nonlinear partial differential equations are useful in describing the various phenomena in many disciplines. Apart of a limited number of these problems, most of them do not have a precise analytical solution, so these nonlinear equations should be solved using approximate methods. In 1992, Shijun Liao employed the basic ideas of the homotopy in topology to propose a general analytic method for nonlinear problems, namely homotopy analysis method (HAM) [1], [2] and then modified it, step by step [3-5]. This method has been successfully applied to solve many types of nonlinear problems by convergence others [6-12]. This method doesn't depend upon any small or large parameters and is valid for most nonlinear models [13]. HAM is different from all previous numerical methods, it contains a certain auxiliary parameter $\mathrm{h}$, which provides us with a simple way to adjust and control the convergence region of solution series [14].The main goal of this paper is to find the approximate solution of the nonlinear diffusion equation with convection term by the homotopy analysis method that has already been successfully applied to several nonlinear problems.

\section{Mathematical model}

The general form of nonlinear diffusion equation with convection and source terms is:

$u_{t}=\left(A(u) u_{x}\right)_{x}+B(u) u_{x}+C(u)$

Where $u=u(x, t)$ is the unknown function and $A(u)$ is the given diffusion coefficient $B(u)$ and $C(u)$ are respectively the convection and source terms they are arbitrary smooth functions on $u$. The indices $\mathrm{t}$ and $\mathrm{x}$ denotes differentiating with respect to the variables. The study of solution of this problem has over the years attracted the interest of many researches. Chemiha and Serov (see [15]) considered the lie and non-lie symmetries of nonlinear diffusion with convection term.

These types of equation arise in several important physical applications including engineering [16], physics [17], the theory of chemical reactions [18], and biology [19].

Taking $A(u)=a, B(u)=b u$, and $C(u)=c u(u-k)(u+k)$ in equation (1), we will get the following model of nonlinear diffusion equation with convection term:

$u_{t}=a u_{x x}+b u u_{x}+c u(u-k)(u+k)$

Subject to the initial condition $u(x, 0)=f(x), \quad \alpha \leq x \leq \beta$

And boundary conditions 
$u(\alpha, t)=g_{1}(t), \quad u(\beta, t)=g_{2}(t), \quad t>0$

Where $a, b, c, k, \alpha$, and $\beta$ are arbitrary constants.

In this article, we will apply homotopy analysis method to approximate solution of the equation (2).

\section{Main idea of homotopy analysis method}

To describe the main ideas of the HAM, we consider the following differential equation: [20]

$N[u(x, t)]=0$,

Where $N$ is a nonlinear operator, $x$ and $t$ denote the independent variables, and $u(x, t)$ is an unknown function. By means of generalizing the traditional homotopy method, Liao (see [1-2]).construct the so-called zeroth-order deformation equation

$(1-p) L\left[\phi(x, t ; p)-u_{0}(x, t)\right]=p \hbar H(x, t) N[\phi(x, t ; p)]$

Where $p \in[0,1]$ denote the so-called embedding parameter, $\hbar \neq 0$ is an auxiliary parameter, and $L$ is an auxiliary linear operator.

The HAM is based on a kind of continuous mapping $u(x, t) \rightarrow \phi(x, t ; p), \phi(x, t ; p)$ is an unknown function, $u_{0}(x, t)$ is an initial guess of $u(x, t)$, and $H(x, t)$ denotes a non-zero auxiliary function. It is obvious that when the embedding parameter $p=0$, and $p=1$ equation (5) becomes:

$\phi(x, t ; 0)=u_{0}(x, t)$,

$\phi(x, t ; 1)=u(x, t)$

respectively. Thus as $p$ increases form 0 to 1 , the solution $\phi(x, t ; p)$ varies from the initial guess $u_{0}(x, t)$ to the solution $u(x, t)$. In topology, this kind of variation is the called deformation, equation (5) construct the homotopy $\phi(x, t ; p)$, and equation (5) is called the zero-deformation equation.

Having the freedom to choose the auxiliary parameter $\hbar$, the auxiliary function $H(x, t)$, the initial approximation $u_{0}(x, t)$, and the auxiliary linear operator $\mathrm{L}$, we can assume that all of them are properly chosen so that the solution $\phi(x, t ; p)$ of the zero-order deformation Equation (5) exists for $0 \leq p \leq 1$.

Expanding $\phi(x, t ; p)$ in the Taylor series with respect to $p$, one has

$\phi(x, t ; p)=u_{0}(x, t)+\sum_{m=1}^{\infty} u_{m}(x, t) p^{m}$,

Where

$u_{m}(x, t)=\left.\frac{1}{m !} \frac{\partial^{m} \phi(x, t ; p)}{\partial p^{m}}\right|_{p=0}$

Assume that the auxiliary parameter $\hbar$, the auxiliary function $H(x, t)$, the initial approximation $u_{0}(x, t)$ and the auxiliary linear operator $\mathrm{L}$ are so properly chosen that the series (7) converges at $p=1$, and

$\phi(x, t ; 1)=u_{0}(x, t)+\sum_{m=1}^{\infty} u_{m}(x, t)$,

Which must be one of the solutions of the original nonlinear equation, as proven by Liao 2009 (see [21]) as $\hbar=-1$ and $H(x, t)=1$ equation $(5)$ becomes:

$(1-p) L\left[\varnothing(x, t ; p)-u_{0}(x, t)\right]+p N[\phi(x, t ; p)]=0$

This is mostly used in the homotopy perturbation method (see [22]).

According to definition (9), the governing equation and the corresponding initial condition of $u_{m}(x, t)$ can be deduced form the zero-deformation equation (5). Define the vectors

$\vec{u}_{n}(x, t)=\left\{u_{0}(x, t), u_{1}(x, t), u_{2}(x, t), \ldots, u_{n}(x, t)\right\}$,

Differentiating equation (5) $m$ times with respect to the embedding parameter $p$ and then dividing them by $m$ ! and finally setting $p=0$, we have the so-called mth-order deformation equation:

$L\left[u_{m}(x, t)-\chi_{m} u_{m-1}(x, t)\right]=\hbar H(x, t) \Re_{m}\left(\vec{u}_{m-1}\right)$,

Where

$\Re_{m}\left(\vec{u}_{m-1}\right)=\left.\frac{1}{(m-1) !} \frac{\partial^{m-1} N[\phi(x, t ; p)]}{\partial p^{m-1}}\right|_{p=0}$

And

$\chi_{m}= \begin{cases}0, & m \leq 1 \\ 1, & m>1\end{cases}$

It should be emphasized that $u_{m}(x, t)$ for $m \geq 1$ is governed by the linear equation (12) with linear boundary conditions that comes from the original problem, which can be easily solved by the symbolic computation software such as Maple, Mathematica and Matlab. 


\section{Approximate solution to the nonlinear diffusion equation with convection term by (HAM)}

To find the approximate solution to the equation (2), according to the style of the solution and the initial condition, we choose the linear operator as

$L[\phi(x, t ; p)]=\frac{\partial \phi(x, t ; p)}{\partial t}$

With the property:

$L[c]=0$

Where $\mathrm{c}$ is constant, and assumes that the inverse $\mathrm{L}_{\mathrm{t}}^{-1}$ exists and is defined as:

$\mathrm{L}_{\mathrm{t}}^{-1}=\int_{0}^{\mathrm{t}}(.) \mathrm{dt}$

Now, we define a nonlinear operator as:

$N[\phi(x, t ; p)]=\frac{\partial \phi(x, t ; p)}{\partial t}-a \frac{\partial^{2} \phi(x, t ; p)}{\partial x^{2}}-b \phi(x, t ; p) \frac{\partial \phi(x, t ; p)}{\partial x}-c \phi(x, t ; p)[(\phi(x, t ; p)-k)(\phi(x, t ; p)+k)]$

Using the above definition, we construct the zeroth-order deformation equation:

$(1-p) L\left[\varnothing(x, t ; p)-u_{0}(x, t)\right]=p \hbar H(x, t) N[\phi(x, t ; p)]$

$p=0$, and $p=1$, we can write

$\phi(x, t ; 0)=u_{0}(x, t), \quad$ and $\phi(x, t ; 1)=u(x, t)$

Thus, we obtain the $m^{\text {th }}$ order deformation equations

$L\left[u_{m}(x, t)-\chi_{m} u_{m-1}(x, t)\right]=\hbar H(x, t) \Re_{m}\left(\vec{u}_{m-1}\right)$

Now, the solution of the $m^{t h}$ order deformation equations are

$u_{m}(x, t)=\chi_{m} u_{m-1}(x, t)+\hbar H(x, t) L^{-1}\left[\mathfrak{R}_{m}\left(\vec{u}_{m-1}\right)\right]$, for $m \geq 1$, and choosing $H(x, t)=1$

$\mathfrak{R}_{m}\left(\vec{u}_{m-1}\right)=\frac{\partial u_{m-1}(x, t)}{\partial t}-a \frac{\partial^{2} u_{m-1}(x, t)}{\partial x^{2}}-b \sum_{i=0}^{m-1} u_{i}(x, t) \frac{\partial u_{m-1-i}(x, t)}{\partial x}$

$$
{ }_{-c}\left(\left[\sum_{i=0}^{m-1} u_{m-1-i}(x, t) \sum_{j=0}^{i} u_{j}(x, t) u_{i-j}(x, t)\right]-k^{2} u_{m-1}(x, t)\right)
$$

Put $m=1$, equation (22) becomes:

$u_{1}(x, t)=\hbar L^{-1}\left[\Re_{1}\left(\vec{u}_{0}\right)\right]$

And equation (23) becomes:

$$
\begin{aligned}
\Re_{1}\left(\vec{u}_{0}\right) & =\frac{\partial u_{0}(x, t)}{\partial t}-a \frac{\partial^{2} u_{0}(x, t)}{\partial x^{2}}-b u_{0}(x, t) \frac{\partial u_{0}(x, t)}{\partial x}-c\left(u_{0}{ }^{3}(x, t)-k^{2} u_{0}(x, t)\right) \\
& =-a \frac{\partial^{2} u_{0}(x, t)}{\partial x^{2}}-b u_{0}(x, t) \frac{\partial u_{0}(x, t)}{\partial x}-c\left(u_{0}{ }^{3}(x, t)-k^{2} u_{0}(x, t)\right)
\end{aligned}
$$

Substituting equations (25) and initial approximation $u_{0}(x, t)$ into equation (24), to obtain a first approximation $u_{1}(x, t)$ as follows:

$$
\begin{aligned}
& u_{1}(x, t)=\hbar \int_{0}^{t}\left(-a \frac{\partial^{2} u_{0}(x, \tau)}{\partial x^{2}}-b u_{0}(x, \tau) \frac{\partial u_{0}(x, \tau)}{\partial x}-c\left(u_{0}{ }^{3}(x, \tau)-k^{2} u_{0}(x, \tau)\right)\right) d \tau \\
& u_{1}(x, t)=-\hbar\left(a \frac{\partial^{2} u_{0}(x, t)}{\partial x^{2}}+b u_{0}(x, t) \frac{\partial u_{0}(x, t)}{\partial x}+c\left(u_{0}{ }^{3}(x, t)-k^{2} u_{0}(x, t)\right)\right) t \\
& \text { Let: } w_{0}(x, t)=a \frac{\partial^{2} u_{0}(x, t)}{\partial x^{2}}+b u_{0}(x, t) \frac{\partial u_{0}(x, t)}{\partial x}+c\left(u_{0}{ }^{3}(x, t)-k^{2} u_{0}(x, t)\right) \\
& \text { so : } u_{1}(x, t)=-\hbar w_{0}(x, t) t
\end{aligned}
$$

Put $m=2$, equation (22) becomes:

$u_{2}(x, t)=u_{1}(x, t)+\hbar L^{-1}\left[\Re_{2}\left(\vec{u}_{1}\right)\right]$

And equation (23) becomes:

$\mathfrak{R}_{2}\left(\vec{u}_{1}\right)=\frac{\partial u_{1}(x, t)}{\partial t}-a \frac{\partial^{2} u_{1}(x, t)}{\partial x^{2}}-b\left(u_{0}(x, t) \frac{\partial u_{1}(x, t)}{\partial x}+u_{1}(x, t) \frac{\partial u_{0}(x, t)}{\partial x}\right)-c\left(3 u_{1}(x, t) u_{0}{ }^{2}(x, t)-k^{2} u_{1}(x, t)\right)$

Substituting equation (28) into equation (30), we get:

$$
\begin{aligned}
\mathfrak{R}_{2}\left(\vec{u}_{1}\right)= & \frac{\partial}{\partial t}\left(-\hbar w_{0}(x, t) t\right)-a \frac{\partial^{2}}{\partial x^{2}}\left(-\hbar w_{0}(x, t) t\right)-b\left(u_{0}(x, t) \frac{\partial}{\partial x}\left(-\hbar w_{0}(x, t) t\right)+\left(-\hbar w_{0}(x, t) t\right) \frac{\partial u_{0}(x, t)}{\partial x}\right) \\
& -c\left(3\left(-\hbar w_{0}(x, t) t\right) u_{0}^{2}(x, t)-k^{2}\left(-\hbar w_{0}(x, t) t\right)\right)
\end{aligned}
$$




$$
\begin{aligned}
\Re_{2}\left(\vec{u}_{1}\right)= & -\hbar w_{0}(x, t)+\hbar\left(a \frac{\partial^{2} w_{0}(x, t)}{\partial x^{2}}+b u_{0}(x, t) \frac{\partial w_{0}(x, t)}{\partial x}+b w_{0}(x, t) \frac{\partial u_{0}(x, t)}{\partial x}+3 c w_{0}(x, t) u_{0}{ }^{2}(x, t)\right. \\
& \left.-c k^{2} w_{0}(x, t)\right) t
\end{aligned}
$$

Substituting equations (28) and (31) in equation (29), to obtain the second order approximation $u_{2}(x, t)$ as follows:

$$
\begin{aligned}
& u_{2}(x, t)=-\hbar w_{0}(x, t) t+\hbar \int_{0}^{t}\left(-\hbar w_{0}(x, \tau)+\hbar\left(a \frac{\partial^{2} w_{0}(x, \tau)}{\partial x^{2}}+b u_{0}(x, t) \frac{\partial w_{0}(x, \tau)}{\partial x}+b w_{0}(x, \tau) \frac{\partial u_{0}(x, \tau)}{\partial x}\right.\right. \\
& \left.\left.+3 c w_{0}(x, \tau) u_{0}^{2}(x, \tau)-c k^{2} w_{0}(x, \tau)\right) \tau\right) d \tau \\
& u_{2}(x, t)=-\hbar w_{0}(x, t) t-\hbar^{2} w_{0}(x, t) t+\hbar^{2}\left(a \frac{\partial^{2} w_{0}(x, t)}{\partial x^{2}}+b u_{0}(x, t) \frac{\partial w_{0}(x, t)}{\partial x}+b w_{0}(x, t) \frac{\partial u_{0}(x, t)}{\partial x}\right. \\
& \left.+3 c u_{0}^{2}(x, \tau) w_{0}(x, t)-c k^{2} w_{0}(x, t)\right) \frac{t^{2}}{2} \\
& \text { Let } w_{1}(x, t)=a \frac{\partial^{2} w_{0}(x, t)}{\partial x^{2}}+b u_{0}(x, t) \frac{\partial w_{0}(x, t)}{\partial x}+b w_{0}(x, t) \frac{\partial u_{0}(x, t)}{\partial x}+3 c u_{0}^{2}(x, \tau) w_{0}(x, t)-c k^{2} w_{0}(x, t) \\
& \text { So } u_{2}(x, t)=\hbar(-1-\hbar) w_{0}(x, t) t+\hbar^{2} w_{1}(x, t) \frac{t^{2}}{2}
\end{aligned}
$$

By the same way we can continue,

Then the approximate solution of equation (2) by HAM is:

$$
u(x, t)=u_{0}(x, t)+\sum_{m=1}^{\infty} u_{m}(x, t)=u_{0}(x, t)+u_{1}(x, t)+u_{2}(x, t)+\cdots
$$

\section{Numerical example}

We will apply homotopy Analysis method (HAM) to solve the nonlinear diffusion equation with convection term, and present numerical results to verify the effectiveness of this method, we take the following example:

Consider the following nonlinear diffusion equation with convection term. [23]

$\frac{\partial u}{\partial t}=a \frac{\partial^{2} u}{\partial x^{2}}+b u \frac{\partial u}{\partial x}+\frac{b^{2}}{9 a} u(u-k)(u+k)$

With the initial condition

$$
u(x, 0)=\frac{k\left(-1+c_{1} e^{\frac{b k x}{3 a}}\right)}{1+c_{1} e^{\frac{b k x}{3 a}}+c_{2} e^{\frac{b k x}{6 a}}}
$$

And boundary conditions

$$
u(0, t)=\frac{k\left(-1+c_{1}\right)}{1+c_{1}+c_{2} e^{\frac{b^{2} k^{2} t}{12 a}}} \text {, and } u(1, t)=\frac{k\left(-1+c_{1} e^{\frac{b k}{3 a}}\right)}{1+c_{1} e^{\frac{b k}{3 a}}+c_{2} e^{\frac{b^{2} k^{2} t}{12 a}+\frac{b k}{6 a}}}
$$

Where $a \neq 0, b, k, c_{1}$ and $c_{2}$ are arbitrary constants.

The exact solution of the above example has been derived by Andrei D. Polyanin and Valentin F. Zaitsev. [23]

$u(x, t)=\frac{k\left(-1+c_{1} e^{\frac{b k x}{3 a}}\right)}{1+c_{1} e^{\frac{b k x}{3 a}}+c_{2} e^{\frac{b^{2} k^{2} t}{12 a}+\frac{b k x}{6 a}}}$

In our work, we used Matlab software for computations $u_{1}(x, t)$ and $u_{2}(x, t)$ and we calculated as follows:

$$
\begin{aligned}
u_{0}(x, t)= & \frac{k\left(-1+c_{1} e^{\frac{b k x}{3 a}}\right)}{1+c_{1} e^{\frac{b k x}{3 a}}+c_{2} e^{\frac{b k x}{6 a}}} \\
u_{1}(x, t)= & \frac{\hbar b^{2} k^{3} t c_{2} e^{\frac{b k x}{6 a}}\left(-1+c_{1} e^{\frac{b k x}{3 a}}\right)}{12 a\left(1+c_{1} e^{\frac{b k x}{3 a}}+c_{2} e^{\left.\frac{b k x}{6 a}\right)^{2}}\right.} \\
u_{2}(x, t)= & \frac{\hbar b^{2} k^{3} t c_{2} e^{\frac{b k x}{6 a}}\left(-1+c_{1} e^{\frac{b k x}{3 a}}\right)}{12 a\left(1+c_{1} e^{\frac{b k x}{3 a}}+c_{2} e^{\left.\frac{b k x}{6 a}\right)^{2}}\right.}+\frac{\hbar^{2} b^{2} c_{2} e^{\frac{b k x}{6 a} k^{3} t\left(-1+c_{1} e^{\frac{b k x}{3 a}}\right)}}{288 a^{2}\left(1+c_{1} e^{\frac{b k x}{3 a}}+c_{2} e^{\left.\frac{b k x}{6 a}\right)^{3}}\right.}\left(24 a-b^{2} k^{2} t+24 a c_{1} e^{\frac{b k x}{3 a}}\right. \\
& \left.\quad+24 a c_{2} e^{\frac{b k x}{6 a}}-b^{2} k^{2} t c_{1} e^{\frac{b k x}{3 a}}+b^{2} k^{2} t c_{2} e^{\frac{b k x}{6 a}}\right)
\end{aligned}
$$

Then the approximate solution of second-order is: 
$u(x, t)=u_{0}(x, t)+\sum_{m=1}^{2} u_{m}(x, t)=u_{0}(x, t)+u_{1}(x, t)+u_{2}(x, t)$

The results obtained by HAM is tabulated in the following tables, followed by their figures and comparisons For $c_{1}=c_{2}=1$ and $a=b=k=0.2$

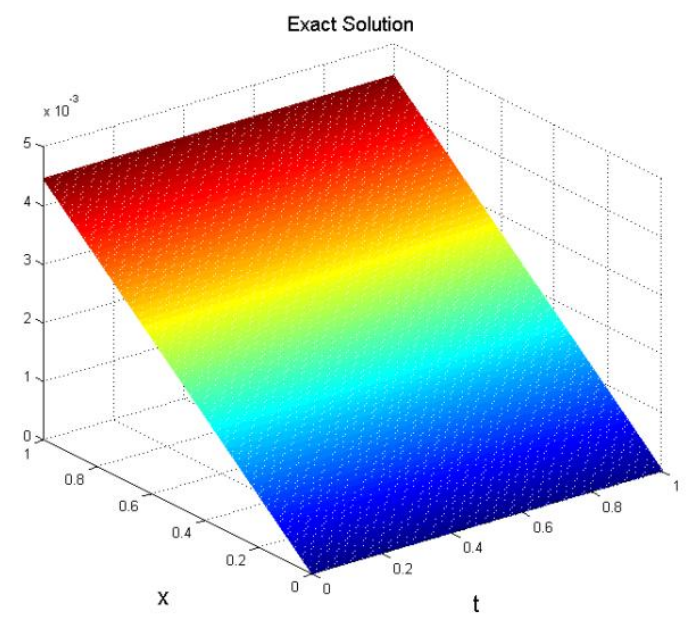

Fig. 1: Shows The Exact Solution

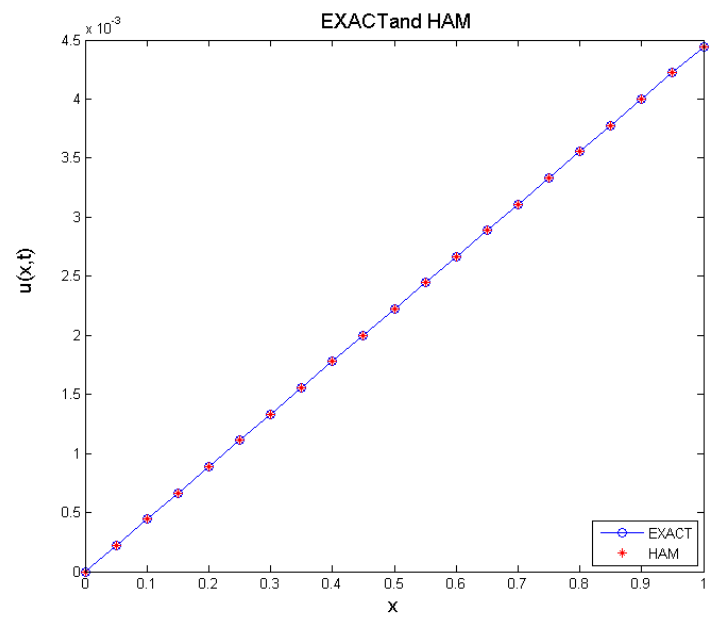

Fig. 3: Shows the Comparison between Exact and (HAM) $t=0.9, \hbar=-1$

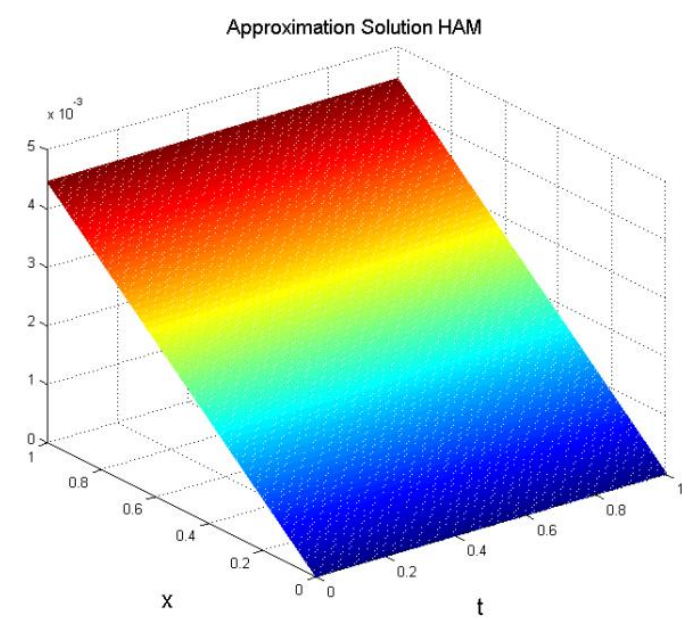

Fig. 2: Shows The (HAM) Solution for $\hbar=-1$

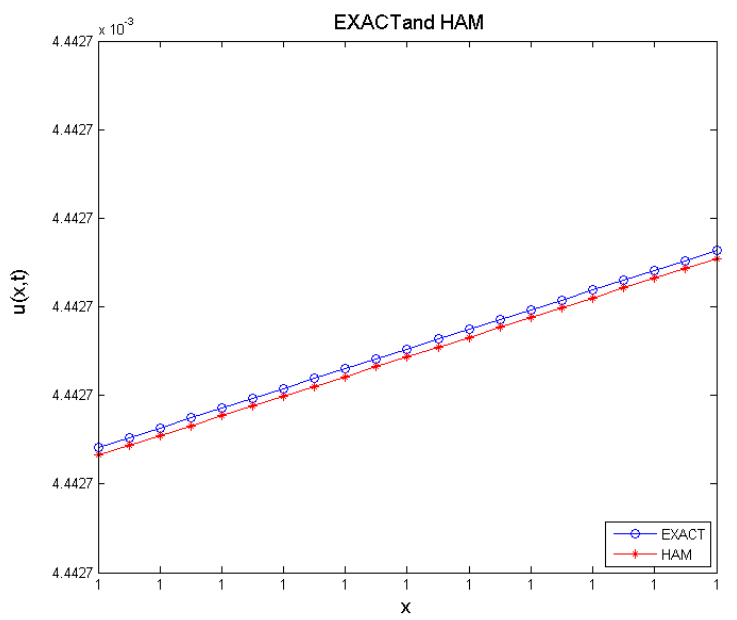

Fig. 4: Zoom For Comparison between Exact and (HAM) Solutions At $0.9999999999 \leq x \leq 1, t=0.9, \hbar=-1$

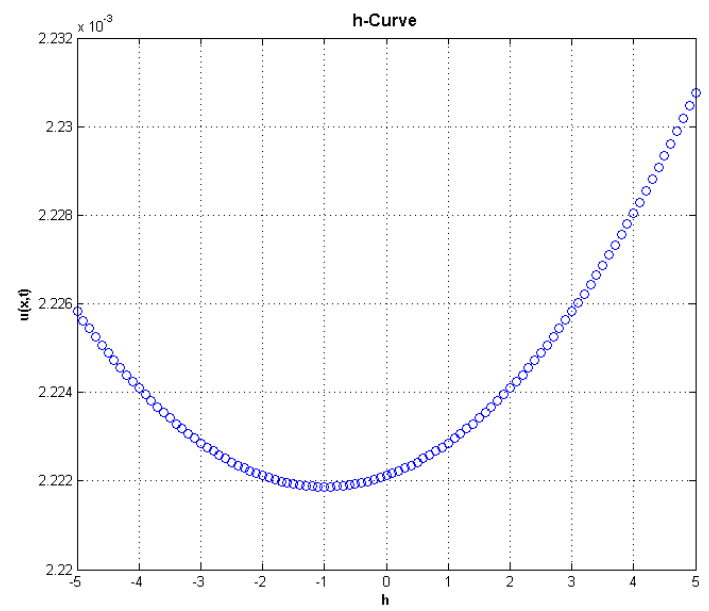

Fig. 5: The $\hbar-$ Curves at $(x, t)=(0.5,0.5)$ 
Table 1: The Results of Exact Solution and (HAM) with Different Values Of $\hbar$, For $0 \leq x \leq 1,0 \leq t \leq 1$

\begin{tabular}{|c|c|c|c|c|c|}
\hline $\begin{array}{l}\text { Space } \\
(x)\end{array}$ & $\begin{array}{c}\text { Time } \\
(t)\end{array}$ & $\begin{array}{c}\text { Exact } \\
\text { Solution }\end{array}$ & $\begin{array}{c}\text { HAM } \\
\hbar=-0.9\end{array}$ & $\begin{array}{c}\text { HAM } \\
\hbar=-1\end{array}$ & $\begin{array}{c}\text { HAM } \\
\hbar=-1.1\end{array}$ \\
\hline \multirow{5}{*}{0.2} & 0 & 0.0008888823045901494534 & 0.0008888823045901492366 & 0.0008888823045901492366 & 0.0008888823045901492366 \\
\hline & 0.2 & 0.0008888427984173288181 & 0.0008888431936370440626 & 0.0008888427984172895700 & 0.0008888431932858754241 \\
\hline & 0.6 & 0.0008887637808050953721 & 0.0008887649674641353919 & 0.0008887637808040415276 & 0.0008887649643036181881 \\
\hline & 0.8 & 0.0008887242693661509367 & 0.0008887258522443317868 & 0.0008887242693636531517 & 0.0008887258466256345475 \\
\hline & 1 & 0.0008886847561723003851 & 0.0008886867356022956241 & 0.0008886847561674218006 & 0.0008886867268230810727 \\
\hline \multirow{5}{*}{0.8} & 0.2 & 0.0035549762492641853034 & 0.0035549778296165108690 & 0.0035549762492640287447 & 0.0035549778282116810575 \\
\hline & 0.4 & 0.0035548182702102681767 & 0.0035548214322485734151 & 0.0035548182702090204768 & 0.0035548214266292524344 \\
\hline & 0.6 & 0.0035546602841340724579 & 0.0035546650291910734248 & 0.0035546602841298609830 & 0.0035546650165476016518 \\
\hline & 0.8 & 0.0035545022910365335969 & 0.0035545086204440113319 & 0.0035545022910265511307 & 0.0035545085979667287099 \\
\hline & 1 & 0.0035543442909185883442 & 0.0035543522060073871362 & 0.0035543442908990896187 & 0.0035543521708866327412 \\
\hline \multirow{5}{*}{1} & 0 & 0.0044436215972679035668 & 0.0044436215972679035668 & 0.0044436215972679035668 & 0.0044436215972679035668 \\
\hline & 0.2 & 0.0044434241717102220676 & 0.0044434261467558751335 & 0.0044434241717100269112 & 0.0044434261449997208837 \\
\hline & 0.4 & 0.0044432267373729385229 & 0.0044432306891314214681 & 0.0044432267373713790065 & 0.0044432306821068044689 \\
\hline & 0.8 & 0.0044428318423642403773 & 0.0044428397525452401756 & 0.0044428318423517677155 & 0.0044428397244467704441 \\
\hline & 1 & 0.0044426343816951667856 & 0.0044426442735835116812 & 0.0044426343816708043291 & 0.0044426442296796528342 \\
\hline
\end{tabular}

Table 2: The Comparison of Solutions Using HAM with the Exact Solution, For $0 \leq x \leq 1,0 \leq t \leq 1, \hbar=-1$

\begin{tabular}{|c|c|c|c|c|}
\hline Space $(x)$ & Time $(t)$ & Exact Solution & HAM Solution & Absolute Error \\
\hline \multirow{6}{*}{0.2} & 0 & 0.0008888823045901494534 & 0.0008888823045901492366 & $2.1684 \mathrm{E}-19$ \\
\hline & 0.2 & 0.0008888427984173288181 & 0.0008888427984172895700 & 3.9248E-17 \\
\hline & 0.4 & 0.0008888032904888992867 & 0.0008888032904885870365 & $3.1225 \mathrm{E}-16$ \\
\hline & 0.6 & 0.0008887637808050953721 & 0.0008887637808040415276 & $1.0538 \mathrm{E}-15$ \\
\hline & 0.8 & 0.0008887242693661509367 & 0.0008887242693636531517 & $2.4978 \mathrm{E}-15$ \\
\hline & 1 & 0.0008886847561723003851 & 0.0008886847561674218006 & $4.8786 \mathrm{E}-15$ \\
\hline \multirow{6}{*}{0.4} & 0 & 0.0017777251049095330656 & 0.0017777251049095328488 & $2.1684 \mathrm{E}-19$ \\
\hline & 0.2 & 0.0017776460978309327216 & 0.0017776460978308544422 & 7.8279E-17 \\
\hline & 0.4 & 0.0017775670872410367397 & 0.0017775670872404124561 & $6.2428 \mathrm{E}-16$ \\
\hline & 0.6 & 0.0017774880731403134952 & 0.0017774880731382066736 & $2.1068 \mathrm{E}-15$ \\
\hline & 0.8 & 0.0017774090555292311466 & 0.0017774090555242368777 & 4.9943E-15 \\
\hline & 1 & 0.0017773300344082587198 & 0.0017773300343985032855 & $9.7554 \mathrm{E}-15$ \\
\hline \multirow{6}{*}{0.6} & 0 & 0.0026664889042949074631 & 0.0026664889042949070294 & 4.3368E-19 \\
\hline & 0.2 & 0.0026663704068429711427 & 0.0026663704068428536152 & $1.1753 \mathrm{E}-16$ \\
\hline & 0.4 & 0.0026662519041238965344 & 0.0026662519041229602174 & 9.3632E-16 \\
\hline & 0.6 & 0.0026661333961383857673 & 0.0026661333961352255348 & $3.1602 \mathrm{E}-15$ \\
\hline & 0.8 & 0.0026660148828871405371 & 0.0026660148828796504349 & $7.4901 \mathrm{E}-15$ \\
\hline & 1 & 0.0026658963643708642743 & 0.0026658963643562344839 & $1.4630 \mathrm{E}-14$ \\
\hline \multirow{6}{*}{0.8} & 0 & 0.0035551342212948870876 & 0.0035551342212948866539 & 4.3368E-19 \\
\hline & 0.2 & 0.0035549762492641853034 & 0.0035549762492640287447 & $1.5656 \mathrm{E}-16$ \\
\hline & 0.4 & 0.0035548182702102681767 & 0.0035548182702090204768 & $1.2477 \mathrm{E}-15$ \\
\hline & 0.6 & 0.0035546602841340724579 & 0.0035546602841298609830 & $4.2115 \mathrm{E}-15$ \\
\hline & 0.8 & 0.0035545022910365335969 & 0.0035545022910265511307 & $9.9825 \mathrm{E}-15$ \\
\hline & 1 & 0.0035543442909185883442 & 0.0035543442908990896187 & $1.9499 \mathrm{E}-14$ \\
\hline \multirow{6}{*}{1} & 0 & 0.0044436215972679035668 & 0.0044436215972679035668 & 0 \\
\hline & 0.2 & 0.0044434241717102220676 & 0.0044434241717100269112 & $1.9516 \mathrm{E}-16$ \\
\hline & 0.4 & 0.0044432267373729385229 & 0.0044432267373713790065 & $1.5595 \mathrm{E}-15$ \\
\hline & 0.6 & 0.0044430292942572212692 & 0.0044430292942519589855 & 5.2623E-15 \\
\hline & 0.8 & 0.0044428318423642403773 & 0.0044428318423517677155 & $1.2473 \mathrm{E}-14$ \\
\hline & 1 & 0.0044426343816951667856 & 0.0044426343816708043291 & $2.4362 \mathrm{E}-14$ \\
\hline
\end{tabular}

\section{Analysis of results}

We first investigate the influence of the auxiliary parameter $\hbar$ on the convergence of the series by plotting the socalled $\hbar$ - curves. By (HAM), it is easy to discover the valid region of $\hbar$, which corresponds to the line segments parallel to the horizontal axis $(\hbar)$.

To determine the valid region of the auxiliary parameter $\hbar$, we plot the $\hbar$ - curves at $(x, t)=(0.5,0.5)$ as shown in Fig. 5, we found that the valid region for $\hbar$ is (-1), i.e. when $\hbar=-1$ we get the best solution of the HAM and it is observed that in Table 1, this fact has been pointed out by Liao [2]. 


\section{Conclusion}

In this paper, the homotopy analysis method has been successfully applied for finding the approximate solutions of the nonlinear diffusion equation with convection term. From the present example, we see that the numerical results and absolute error $\left|u_{\text {Exact }}-u_{H A M}\right|$, the comparison between the (HAM) and the exact solution was made and it was found that (HAM) is closed to the exact solution, very accurate and effective as illustrated in Table 2 and Figs. 1-4 .

The (HAM) contains a certain auxiliary parameter which provides us with a simple way to adjust and control the convergence region and rate of convergence of the series solution as shown in Fig. 5. Hence, it may be concluded that (HAM) is powerful mathematical tool for solving nonlinear problems in science and engineering.

\section{Acknowledgements}

The authors thank the editor and the referees for their comments and suggestions to improve the paper.

\section{References}

[1] S.J. Liao," The proposed homotopy analysis method techniques for the solution of nonlinear problems", Thesis Shanghai, Jiao Tong University Shanghai, (1992).

[2] S.J. Liao," Beyond Perturbation: Introduction to the Homotopy Analysis Method", Chapman and Hall/CRC Press, Boca Raton, (2003).

[3] J.D. Cole," Perturbation Method in Applied Mathematics", Blaisdell, Waltham, MA, (1968).

[4] J.H. He," Homotopy perturbation technique", Comput. Methods Appl, Mech, Engrg, 178, (1999), $257-262$.

[5] S.J. Liao," On the homotopy analysis method for nonlinear problems", App. Math. Comput. 147, (2004), $499-513$.

[6] M. Ayub,A. Rasheed,T. Hayat," Exact flow of a third grade fluid past a porous plate using homotopy analysis”, Int. J. Eng. Sci, 41, (2003), 2091-2103.

[7] T. Hayat,M. Khan," Exact flow of a third grade fluid past a porous plate using homotopy analysis", Int. J. Eng. Sci, 56, (2005), 1012-1029.

[8] M. Hayat,M. Khan," Homotopy solutions for a generalized second grade fluid past a porous plate", Nonlinear Dynam, 42, (2005), 395-405.

[9] M. Sajad,T. Hayat,S. Asghar," On the analytic solution of the steady flow of a fourth grade fluid. Phys.”, Lett. A, 355, (2006), 18-26.

[10] B. Raftari," Application of He's homotopy perturbation method and variational iteration method for nonlinear partial integro-differential equations", World Applied Sciences Journal, 7, 4, (2009), 399-404.

[11] B. Raftari," Numerical solutions of the linear volterra integro-differential equations: homotopy perturbation method and finite Difference method", World Applied Sciences Journal, 9, (2010), 7-12.

[12] M. Matinfar,M. Saeidy," The homotopy perturbation method for solving higher dimensional initial boundary value problems of variable coefficients", World Applied Sciences Journal, 5, 1, (2009),72-80.

[13] Y. Mahmoudi,E.M. Kazemian," The Homotopy Analysis Method for Solving the Kuramoto-Tsuzuki Equation ",World Applied Sciences Journal, 21, 12, (2013), 1776-1781.

[14] Ch. Xiurong,Yu. Jiaju," Homotopy Analysis Method for a Class of Holling Model with the Functional Reaction”, the Open Automation and Control Systems Journal, 5, (2013), 150-153.

[15] R. Cherniha,M. Servo," Lie and non-lie symmetries of nonlinear di_usion equations with convection term ", Symmetry in Nonlinear Mathematical Physics, 2, (1997), 444-449.

[16] W.F. Ames,” Nonlinear Partial Differential Equations in Engineering “, Academic Press, New York, (1972).

[17] J.Crank," The Mathematics of Diffusion ", Oxford University Press, Oxford, (1975).

[18] R. Aris," The Mathematical Theory of Diffusion and Reaction in Permeable Catalysts “, Oxford University Press, Oxford, (1975).

[19] J.D. Murray," Mathematical Biology “, Springer, Berlin, (1989).

[20] M.A. El-Tawi,H.N.Hassan," A new application of using homotopy analysis method for solving stochastic quadratic nonlinear diffusion equation "Int. J. of Appl Math and Mech, 9, 16, (2013), 35-55.

[21] S.J. Liao," Notes on the homotopy analysis method: some definitions and theories ", Communications in Nonlinear Science Numerical Simulation, 14, (2009), 983-997.

[22] M. Sajid,T. Hayat,S. Asghar," Non-similar solution for the ax symmetric flow of a third-grade fluid over radially stretching sheet ", Acta Mechanica, 189, (2007), 193-205.

[23] V.F. Zaitsev,A. Polyanim,” Handbook of Nonlinear Partial Di_erential Equations “, Chapman and Hall/CRC Press, Boca Raton, (2004). 